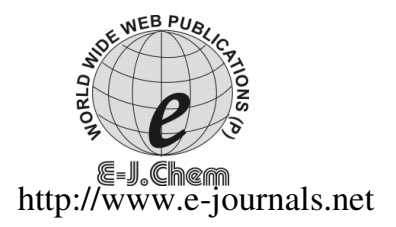

ISSN: 0973-4945; CODEN ECJHAO

E-Journal of Chemistry

2011, 8(3), 1401-1407

\title{
Novel Aryl Ether Derivatives as Antiinflammatory and Analgesics
}

\author{
P. RASHMI ${ }^{*}$, GOPAL KRISHNA RAO, KSHAMA DEVI ${ }^{\S}$, \\ B.G. SHIVANANDA ${ }^{\S}$, G. R. SWETHA and SUNEETHA GA ${ }^{\S}$ \\ *Department of Pharmaceutical Chemistry \\ ${ }^{\S}$ Department of Pharmacology Al-Ameen College of Pharmacy \\ Hosur Road, Bangalore-560027, India \\ Nargund College of Pharmacy \\ II Main Dattatreyanagar, Bangalore-560085, India \\ rshvsh@gmail.com
}

Received 26 September 2010; Accepted 27 November 2010

\begin{abstract}
The diaryl ether moieties have attracted considerable attention of medicinal chemists as they are endowed with a wide range of diverse biological activities. The present study involves synthesis, characterization of some new aryl ethers and evaluation of their antiinflammatory and analgesic activity. A series of new aryl ether derivatives $[\mathbf{4}(\mathbf{a}-\mathbf{h}), \mathbf{5}]$ were prepared by Ullmann's ether condensation. The structures of new compounds are supported by their IR, ${ }^{1} \mathrm{H}$ NMR and Mass spectra. The new derivatives were evaluated for their antiinflammatory and analgesic activity. Among the tested, compound $\mathbf{3}$ has shown better antiinflammatory and analgesic activity.
\end{abstract}

Keywords: Aryl ethers, Antiinflammatory activity, Analgesic activity.

\section{Introduction}

Classical NSAIDs are nonselective inhibitors of COX. Their long term use leads to ulceration. After the discovery of COX-2, its selective inhibition is considered as an alternative pharmacotherapeutic approach ${ }^{1}$. The development of preferential COX-2 inhibitors has been considered as a challenging task to improve benefit/risk ratio of NSAIDs. The diaryl or arylheteroaryl ethers are also known to be selective inhibitors ${ }^{2}$ of COX-2.

Nimesulide $^{3}$ an antiinflammatory agent with an aryl ether linkage is a preferential COX-2 inhibitor. But nimesulide induced liver injury gained investigative interest and was found to cause hepatocellular necrosis or cholestasis ${ }^{4}$. So there is a need for synthesis of new derivatives which are as effective as nimesulide, overcoming its side effects. So the objective of present project is to synthesize and characterize some new aryl ethers and evaluate their antiinflammatory and analgesic activity. 


\section{Experimental}

The IR spectra of the synthesized compounds were recorded on a fourier transform IR spectrometer (model Shimadzu 8700). ${ }^{1} \mathrm{H}$ NMR spectra were recorded on DMM X - 200 MHz NMR, Bruker Daltonics, Karsruhe, Germany. Mass spectra were recorded on mass spectrophotometer (model Shimadzu) by LC-MS. Merck GF254 pre-coated silica plates were used for TLC. The melting points are uncorrected.

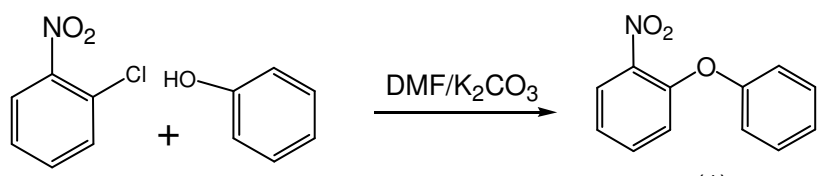

(1)<smiles>[R]CC(=O)Nc1ccccc1Oc1ccccc1</smiles>

(4a-4h)<smiles>Nc1ccccc1Oc1ccccc1</smiles>

(2)$$
\text { (1) }
$$

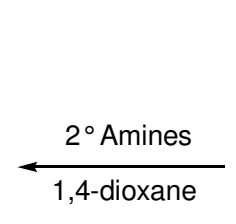<smiles>O=C(CCl)Nc1ccccc1Oc1ccccc1</smiles>

(3)<smiles>CC(C)Cl</smiles>

(2)

Scheme 1<smiles>O=C(Cl)c1ccccc1</smiles><smiles>O=C(Nc1ccccc1Oc1ccccc1)c1ccccc1</smiles>

(5)

\section{Scheme 2}

\section{Preparation $^{5}$ of o-phenoxynitrobenzene (1)}

To a solution of $o$-chloronitrobenzene $(0.15 \mathrm{M})$ in DMF, equimolar quantities of $\mathrm{K}_{2} \mathrm{CO}_{3}$ and phenol were discharged and refluxed with stirring for $8 \mathrm{~h}$. After completion added water, then extracted with methylene chloride which was distilled off to get yellow liquid product. $\%$ Yield: $86.5 \%$, boiling point: $237^{\circ} \mathrm{C}$.

\section{Preparation of o-phenoxyaniline (2)}

$o$-Phenoxynitrobenzene $(0.05 \mathrm{M})$ in ethanol $(50 \% \mathrm{w} / \mathrm{w})$ was subjected to reduction with iron $(0.05 \mathrm{M})$ and dil. $\mathrm{HCl}$ [in $10 \mathrm{~mL}$ of $50 \% \mathrm{w} / \mathrm{w}$ ethanol]. After addition, stirred for another $1 \mathrm{~h}$, cooled and added $15 \% \mathrm{KOH}$ in pure ethanol. Filtered, concentrated and mother liquor cooled in ice. Crystals formed were recrystallised from hexane. \% Yield: 54.05\%, melting point: $256{ }^{0} \mathrm{C}$.

\section{Preparation ${ }^{6}$ of 2-chloro- $N$-(2-phenoxyphenyl)acetamides (3)}

To ice cold solution of $o$-phenoxyaniline $(0.01 \mathrm{M})$ in dry benzene added chloroacetylchloride $(0.05 \mathrm{M})$ in benzene drop wise with stirring. The reaction mixture refluxed for $4 \mathrm{~h}$ and benzene distilled off. The residue was extracted with petroleum ether and evaporated to get yellow solid. \% Yield $85.25 \%$, Melting point: $64{ }^{\circ} \mathrm{C}$. 
$\operatorname{IR}(\mathrm{KBr}) \mathrm{cm}^{-1}: 3366.79$ (NH str.), $1695.31\left(\mathrm{C}=\mathrm{O}\right.$ str), 1604.66, ${ }^{1} \mathrm{H}$ NMR $\left(\mathrm{CDCl}_{3}\right) \delta$ :$8.90\{\mathrm{~s}, \mathrm{NH}(1 \mathrm{H})\}, 7.37-6.88\{\mathrm{~m}, \operatorname{Ar}-\mathrm{H}(9 \mathrm{H})\}, 2.17-2.14\left\{\mathrm{~d}, \mathrm{CH}_{2}(2 \mathrm{H})\right\}, 589.23(\mathrm{C}=\mathrm{C}$ Ar str.), 1319.22 (C-N str.), 1217.00 (C-O-C str). : Base peak (262), $\mathrm{M}^{+}(262), \mathrm{M}+1$ (263), M+2 (264.).

General procedure ${ }^{7}$ for the preparation of 2-substituted-N-(2-phenoxyphenyl) acetamides (4a-4h)

The compound (3) $[(0.01 \mathrm{M})]$, different secondary amines $[(0.03 \mathrm{M})]$ and freshly distilled 1 , 4-dioxan were heated for $4 \mathrm{~h}$ at $50{ }^{\circ} \mathrm{C}$ to $100{ }^{\circ} \mathrm{C}$ with occasional swirling. The reaction mixture was added to ice cold water. The contents were cooled in ice; precipitate was filtered and crystallized from aqueous ethanol.

\section{Spectral data of selected compounds}

\section{2-Morpholin-4-yl-N-(2-phenoxyphenyl)acetamide (4a)}

$\operatorname{IR}(\mathrm{KBr}) \mathrm{cm}^{-1}: 3255.62$ (NH str.), 1703.03 (C=O str), 1585.32, 1531.37 (C=C Ar str.), 1263.6 (C-N str.), 1218.93 (C-O-C str). ${ }^{1} \mathrm{H}$ NMR $\left(\mathrm{CDCl}_{3}\right) \delta:-9.70\{\mathrm{~s}, \mathrm{NH}(1 \mathrm{H})\}, 7.3-6.9$ $\{\mathrm{m}, \mathrm{Ar}-\mathrm{H}(9 \mathrm{H})\}, 3.5-3.4\left\{\mathrm{t}, \mathrm{OCH}_{2}(4 \mathrm{H})\right\}$ 3.1-3.0 $\left\{\mathrm{s}, \mathrm{Ali} \mathrm{CH}_{2}(2 \mathrm{H})\right\}, 2.5-2.4\left\{\mathrm{t}, \mathrm{N}_{-} \mathrm{CH}_{2}(4 \mathrm{H})\right\}$. Mass : Base peak (313), $\mathrm{M}^{+}$(313).

$N$-(2-phenoxyphenyl)-2-piperazin-1-ylacetamide (4b)

$\operatorname{IR}(\mathrm{KBr}) \mathrm{cm}^{-1}: 3286.48$ (NH str.), 3263.33 (ring NH1 str), 1681.81 (C=O str), 1587.31, 1531.37 (C=C Ar str.), 1249.79 (C-N str.), 1215.07 (C-O-C str). ${ }^{1} \mathrm{H}$ NMR $\left(\mathrm{CDCl}_{3}\right)$ $\delta$ :- $10.01\{\mathrm{~s} \mathrm{NH}$-piperazine ring $(1 \mathrm{H})\}, 8.5\{\mathrm{~s}, \mathrm{NH}(1 \mathrm{H})\}, 7.3-6.9\{\mathrm{~m}, \mathrm{Ar}-\mathrm{H}(9 \mathrm{H})\}, 3.25\{\mathrm{~s}$, $\left.\mathrm{CH}_{2}(2 \mathrm{H})\right\}, 2.8-2.6\left\{\mathrm{t}, \mathrm{NH}-\mathrm{CH}_{2}(4 \mathrm{H})\right\}, 2.5-2.4\left\{\mathrm{t}, \mathrm{N}-\mathrm{CH}_{2}(4 \mathrm{H})\right\}$. Base peak (312), $\mathrm{M}+1$ (312), M+2 (313).

\section{2-(Dimethylamino)-N-(2-phenoxyphenyl)acetamides (4c)}

$\operatorname{IR}(\mathrm{KBr}) \mathrm{cm}^{-1}: 3391.77$ (NH str.), 1691.06 (C=O str), 1602.74, 1560.08 (C=C Ar str.), 1317.29 (C-N str.), 1215.07 (C-O-C str). ${ }^{1} \mathrm{H}$ NMR $\left(\mathrm{CDCl}_{3}\right) \delta:-8.9\{\mathrm{~s}, \mathrm{NH}(1 \mathrm{H})\}, 7.4-6.6$ $\{\mathrm{m}, \operatorname{Ar}-\mathrm{H}(9 \mathrm{H})\}, 4.2-4.0\left\{\mathrm{~s}, \mathrm{CH}_{2}(2 \mathrm{H})\right\}, 1.6-1.2\left\{\mathrm{~s}, 2 \mathrm{CH}_{3}(6 \mathrm{H})\right\}$.

\section{2-(1H-Imidazol-1-yl)-N-(2-phenoxyphenyl)acetamides (4e)}

$\operatorname{IR}(\mathrm{KBr}) \mathrm{cm}^{-1}: 3217.04$ (NH str.), 1701.00 (C=O str), 1606.59, 1546.80(C=C Ar str.), 1301.86 (C-N str.), 1211.21 (C-O-C str). ${ }^{1} \mathrm{H}$ NMR $\left(\mathrm{CDCl}_{3}\right) \delta:-9.84\{\mathrm{~s}, \mathrm{NH}(1 \mathrm{H})\}$, 7.59-6.6 m, Ar-H (12H) \}, $4.9\left\{\mathrm{~s}, \mathrm{CH}_{2}(2 \mathrm{H})\right\} .2 .2-1.5\left\{\mathrm{~m}, 2 \mathrm{CH}_{2}(4 \mathrm{H})\right\}, 1.4\left\{\mathrm{t}, 2 \mathrm{CH}_{3}\right.$ $(6 \mathrm{H})$ \}. 1101.3 (Ar-Cl), 3.2 - solvent impurity. Mass : Base peak (294), $\mathrm{M}^{+}$(294), $\mathrm{M}+1$ (295).

\section{(1-\{2-Oxo-2-[(2-phenoxyphenyl)amino]ethyl\}-1H-indol-3-yl) acetic acid (4f)}

$\operatorname{IR}(\mathrm{KBr}) \mathrm{cm}^{-1}: 3390.63$ (OH str), 3134.11 (NH str.), 1703.03 (C=O str), 1606.59, 1537. 16(C=C Ar str.), 1303.79 (C-N str.), 1217. (C-O-C str). ${ }^{1} \mathrm{H}$ NMR $\left(\mathrm{CDCl}_{3}\right) \delta:-12.13$ $\{\mathrm{s}, \mathrm{COOH}(1 \mathrm{H})\}, 10.89\{\mathrm{~s}, \mathrm{NH}(1 \mathrm{H})\}, 8.05-6.6\{\mathrm{~m}, \mathrm{Ar}-\mathrm{H}(14 \mathrm{H})\}, 4.3\left\{\mathrm{~s}, \mathrm{CH}_{2}(2 \mathrm{H})\right\}, 3.3$ $\left\{\mathrm{s}, \mathrm{CH}_{2}\right.$ of $\left.\mathrm{CH}_{2} \mathrm{COOH}(2 \mathrm{H})\right\}$.

\section{Preparation of N-(2-phenoxyphenyl)benzamide (5)}

To ice cold solution of $o$-phenoxyaniline $(0.01 \mathrm{M})$ in dry benzene added benzoylchloride $(0.01 \mathrm{M})$ in benzene with stirring. Stirring continued for half an hour. 
The reaction mixture refluxed for $4 \mathrm{~h}$ and benzene distilled off. The residue was extracted with petroleum ether and evaporated to get solid. $\mathrm{IR}(\mathrm{KBr}) \mathrm{cm}^{-1}: 3230.54(\mathrm{NH}$ str.), 1656.74 ( $\mathrm{C}=\mathrm{O}$ str), 1587.31, 1525.59 (C=C Ar str.), 1217.00 (C-O-C str). Base peak (290), $\mathbf{M}^{+}$(290), $\mathrm{M}+1$ (291).

Table 1. Physicochemical properties<smiles>[R]CC(=O)Nc1ccccc1Oc1ccccc1</smiles>

\begin{tabular}{cccc}
\hline Compound code & $\mathrm{R}$ & \% Yield & Melting point, ${ }^{0} \mathrm{C}$ \\
\hline $\mathbf{4 a}$ & Morpholine & 47.9 & 118 \\
$\mathbf{4 b}$ & Piperazine & 63.05 & 94 \\
$\mathbf{4 c}$ & $-\mathrm{N}\left(\mathrm{CH}_{3}\right)$ & 54.81 & 78 \\
$\mathbf{4 d}$ & $-\mathrm{N}\left(\mathrm{C}_{2} \mathrm{H}_{5}\right)$ & 43.34 & 88 \\
$\mathbf{4 e}$ & Imidazole & 38.7 & 184 \\
$\mathbf{4 f}$ & Indole-3-acetic acid & 56.43 & 148 \\
$\mathbf{4 g}$ & $-\mathrm{NC}_{4} \mathrm{H}_{10}$ & 61.23 & 210 \\
$\mathbf{4 h}$ & $-\mathrm{NHNH}_{2}$ & 56.33 & 78 \\
$\mathbf{5}$ & $-\mathrm{COC}_{6} \mathrm{H}_{5}$ & 44.86 & 114 \\
\hline
\end{tabular}

\section{Pharmacological studies}

In the present study we evaluated the analgesic and anti-inflammatory activities of the synthesized compounds and nimesulide was standard. A simple technique to evaluate inflammatory pain along with anti-inflammatory studies in carrageenan-induced paw edema developed by Jain et al. ${ }^{5-7}$ was used.

Percentage protection was calculated by using the formula, $\%$ protection $=(1-\mathrm{Vt} / \mathrm{Vc})$ $\mathrm{X} 100$, where $\mathrm{Vt}$ is the mean increase in the paw volume in the test group. $\mathrm{Vc}$ is the mean increase in the paw volume in the control group.

\section{Results and Discussion}

In the present work, series of new analogues of diaryl ethers were synthesized and the structures were supported by their spectra. Of the ten synthesized, eight were $(\mathbf{3}, \mathbf{4}(\mathbf{a}-\mathbf{e})$, $4 \mathrm{~g}$ and $4 \mathrm{~h})$ were subjected to evaluation.

\section{Anti-inflammatory activity and analgesic activity}

Nimesulide shown onset antiinflammatory action at $90 \mathrm{~min}$. It exhibited significant antiinflammatory activity at $120 \mathrm{~min}$ and retained the same significance level even at $180 \mathrm{~min}$. The onset of analgesic action was by 30 min, maximum activity was between 90 and $120 \mathrm{~min}$ and showed decline in the activity by $180 \mathrm{~min}$.

The compound (3) exhibited onset of action and significant anti-inflammatory activity at $120 \mathrm{~min}$. Ad ecline in the activity at $180 \mathrm{~min}$ indicated its short duration of action. 
It has shown onset at $60 \mathrm{~min}$ and significant analgesic activity from $90 \mathrm{~min}$ to $120 \mathrm{~min}$. Compared to the standard the potency of compound (3) was found to be less.

Substitutions to $\Omega$ chlorine of compound (3) have not augmented the anti inflammatory and analgesic potency significantly. (4b) with piperazine substitution to (3) also exhibited similar analgesic activity. Imidazole substitution (4e) exhibited early onset of action when compared to compound (3).

The results are shown in Table $2 \& 3$ and Figures $1,2,3 \& 4$.

Table 2. Antiinflammatory activity

\begin{tabular}{cccc}
\hline Groups & $90 \mathrm{~min}$ & $120 \mathrm{~min}$ & $180 \mathrm{~min}$ \\
\hline Control & $14.58 \pm 0.49$ & $20.83 \pm 0.47$ & $18.67 \pm 0.77$ \\
Nimesulide & $11.33 \pm 0.55^{* *}$ & $7.67 \pm 0.21^{* * *}$ & $13.33 \pm 0.42^{* * *}$ \\
$\mathbf{3}$ & $13 \pm 0.57 \mathrm{~ns}$ & $14.17 \pm 0.47^{* * *}$ & $15.50 \pm 0.76^{*}$ \\
$\mathbf{4 a}$ & $13.67 \pm 0.42 \mathrm{~ns}$ & $14.83 \pm 0.47^{* * *}$ & $16.17 \pm 0.30^{*}$ \\
$\mathbf{4 b}$ & $13 \pm 0.51 \mathrm{~ns}$ & $13.83 \pm 0.40^{* * *}$ & $15.67 \pm 0.21^{* *}$ \\
$\mathbf{4 c}$ & $13 \pm 0.36 \mathrm{~ns}$ & $15.00 \pm 0.36^{* * *}$ & $15.50 \pm 0.61^{* *}$ \\
$\mathbf{4 d}$ & $15.67 \pm 0.21 \mathrm{~ns}$ & $15.33 \pm 0.33^{* * *}$ & $15.83 \pm 0.47^{*}$ \\
$\mathbf{4 e}$ & $14.17 \pm 0.16 \mathrm{~ns}$ & $14.67 \pm 0.49^{* * *}$ & $16.00 \pm 0.25^{* *}$ \\
$\mathbf{4 g}$ & $14.83 \pm 0.40 \mathrm{~ns}$ & $15.67 \pm 0.21^{* *}$ & $16.50 \pm 0.56^{*}$ \\
$\mathbf{4 h}$ & $13.17 \pm 0.16 \mathrm{~ns}$ & $14.33 \pm 0.33^{* * *}$ & $15.33 \pm 0.21^{* *}$ \\
F value & $7.96^{* * *}$ & $6.97^{* * *}$ & $6.73^{* * *}$ \\
\hline \multicolumn{4}{c}{$\rightarrow$ non significant, $\rightarrow p<0.05,{ }^{* *} \rightarrow p<0.01,{ }^{* * *} \rightarrow p<0.001$}
\end{tabular}

Table 3. Analgesic activity

\begin{tabular}{|c|c|c|c|c|}
\hline Groups & $60 \mathrm{~min}$ & $90 \mathrm{~min}$ & $120 \mathrm{~min}$ & $180 \min$ \\
\hline Control & $5.33 \pm 0.84$ & $3.83 \pm 1.88$ & $3.94 \pm 0.26$ & $1.78 \pm 0.26$ \\
\hline Nimesulide & $9.33 \pm 0.44^{* *}$ & $9.14 \pm 0.82^{* * *}$ & $9.17 \pm 0.89^{* * * *}$ & $4.03 \pm 0.73^{*}$ \\
\hline 3 & $6.94 \pm 1.66^{*}$ & $6.81 \pm 0.33^{* *}$ & $6.33 \pm 1.31^{* *}$ & $4.17 \pm 1.45^{*}$ \\
\hline $4 a$ & $7.44 \pm 0.32^{*}$ & $6.50 \pm 0.28^{*}$ & $6.27 \pm 0.56^{* *}$ & $3.78 \pm 0.22^{*}$ \\
\hline $4 b$ & $8.50 \pm 0.35^{*}$ & $7.89 \pm 0.22^{* *}$ & $6.17 \pm 0.44^{* *}$ & $3.39 \pm 0.49^{*}$ \\
\hline $4 c$ & $6.39 \pm 0.49 \mathrm{~ns}$ & $4.56 \pm 0.81 \mathrm{~ns}$ & $4.28 \pm 0.67 \mathrm{~ns}$ & $3.78 \pm 0.42^{*}$ \\
\hline $4 d$ & $8.83 \pm 1.20^{*}$ & $6.20 \pm 0.43^{*}$ & $6.18 \pm 0.29^{* *}$ & $2.11 \pm 0.22 \mathrm{~ns}$ \\
\hline $4 e$ & $7.39 \pm 0.26^{*}$ & $6.98 \pm 0.30^{* *}$ & $6.17 \pm 0.11^{* *}$ & $3.28 \pm 0.10^{*}$ \\
\hline $4 g$ & $6.39 \pm 0.21 \mathrm{~ns}$ & $5.22 \pm 0.14 \mathrm{~ns}$ & $4.22 \pm 0.22 \mathrm{~ns}$ & $3.33 \pm 0.86^{*}$ \\
\hline $4 h$ & $6.22 \pm 0.49 \mathrm{~ns}$ & $5.44 \pm 0.07 \mathrm{~ns}$ & $5.14 \pm 0.11 \mathrm{~ns}$ & $3.85 \pm 0.37^{*}$ \\
\hline F value & $8.07^{* * * *}$ & $3.88^{* * * *}$ & $4.35^{* * * *}$ & $4.43^{* * *}$ \\
\hline
\end{tabular}




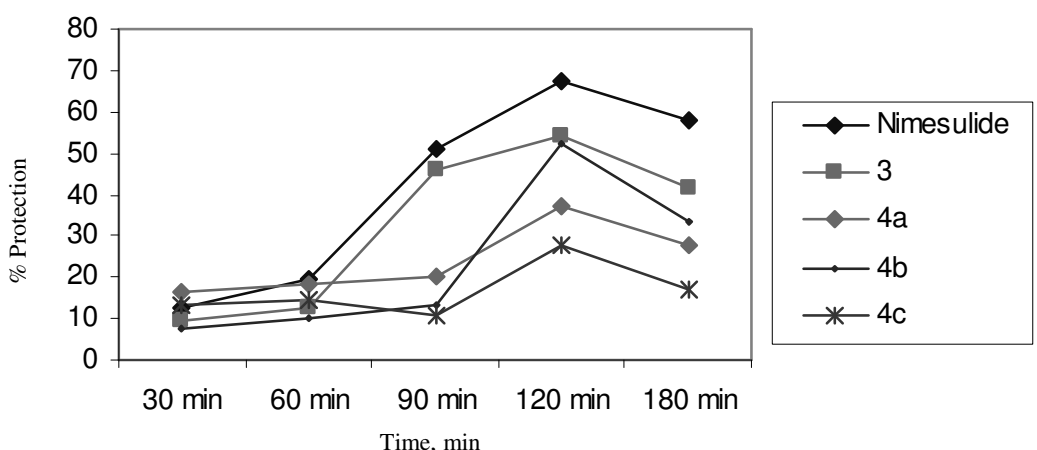

Figure 1. Graphical comparison of antiinflammatory activity

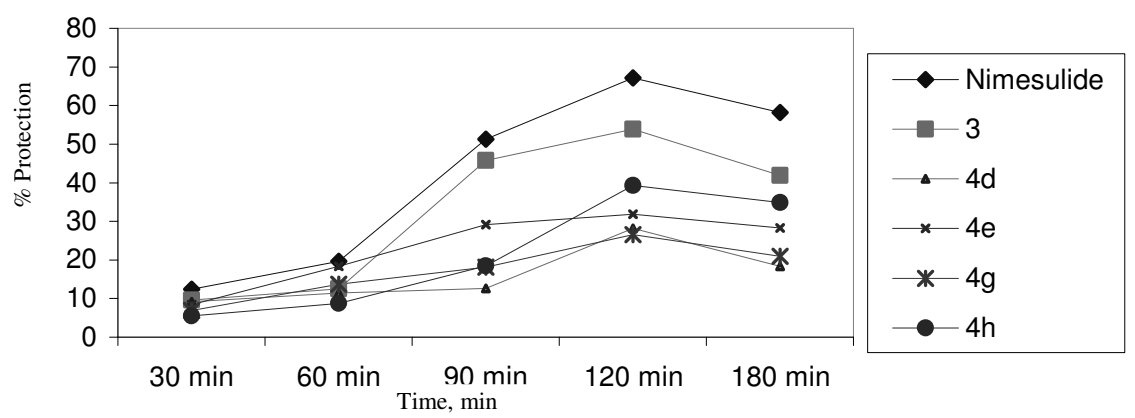

Figure 2. Graphical comparison of antiinflammatory activity

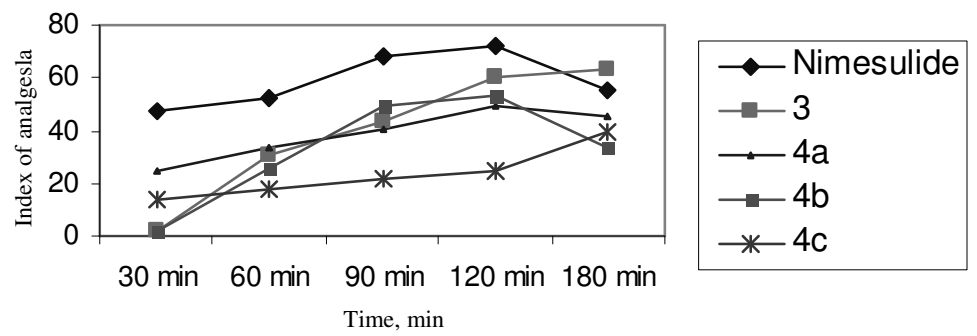

Figure 3. Graphical comparison of analgesic activity

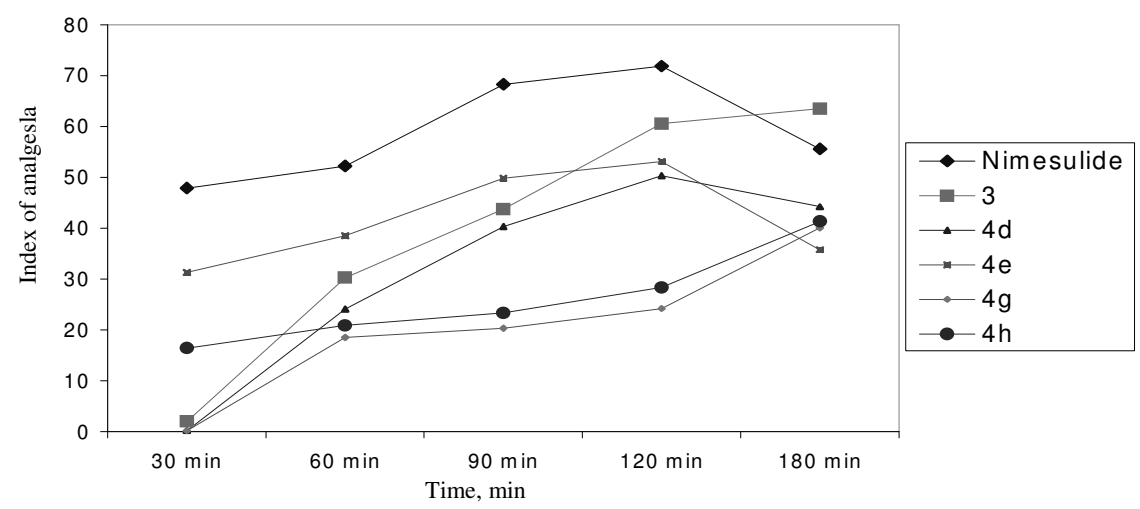

Figure 4. Graphical comparison of analgesic activity 


\section{Conclusion}

The main focus of this research work was to synthesize, purify, characterize and evaluate pharmacological activities of the newly synthesized aryl ethers. A novel and single method was used to perform both the anti-inflammatory and analgesic activities using the rat paw edema method. Most of the compounds tested showed good anti-inflammatory and analgesic activity at the dose of $20 \mathrm{mg} / \mathrm{kg}$.

\section{Acknowledgment}

The author expresses his sincere gratitude to Al-Ameen College of Pharmacy, Bangalore, for their kind help and guidance. Dr.K.S.Bhide, Director R.\& D, Astra-Zeneca Pharma India Ltd., for providing ${ }^{1} \mathrm{H}-\mathrm{NMR}$ spectra and to members of Quest, Bangalore for providing Mass spectra of the synthesized compounds.

\section{References}

1. Leval X, Delarge J, Somers F, Tullio P, Henrotin Y, Pirotte B and Dogne J M, Curr Med Chem., 2000, 7, 1041-1062.

2. Pericherla S, Mareddy J, Rani G D P, Gollapudi P V and Pal S, J Braz Chem Soc., 2007, 18(2), 384-390.

3. Kulkarni S K, Curr Sci., 2002, 83(12), 1442-1443.

4. Thawani V, Sontakke S, Gharpure K and Pimpalkhute S, Indian J Pharm., 2003, 35, 121-122.

5. Gupta S K, Bansal P, Bharadwaj R K and Velpandian T, Pharmacol Res., 2000, 41(6), 657-662.

6. Fattore L, Melis M, Diana M, Fratta W and Gessa G, Eur J Pharmacol., 2000, 406(1), 75-77.

7. Jain N K, Patil C S, Sing A and Kulkarni S K, Indian J Pharmacol., 2001, 33, 114-115. 


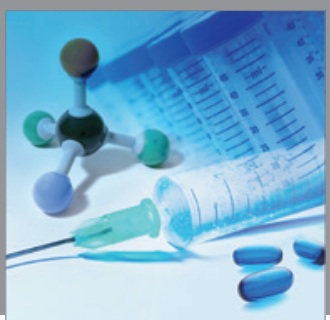

International Journal of

Medicinal Chemistry

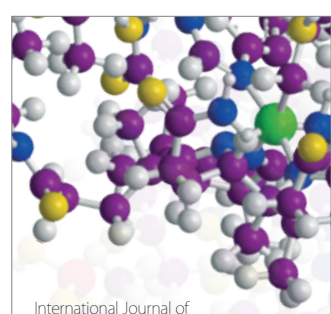

Carbohydrate Chemistry

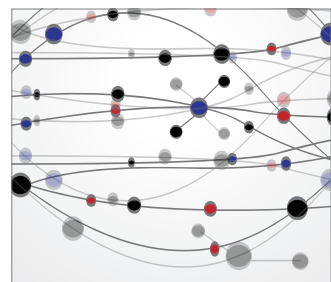

The Scientific World Journal
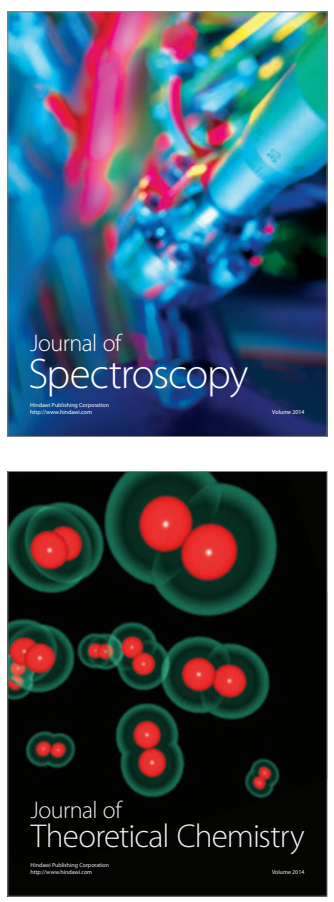
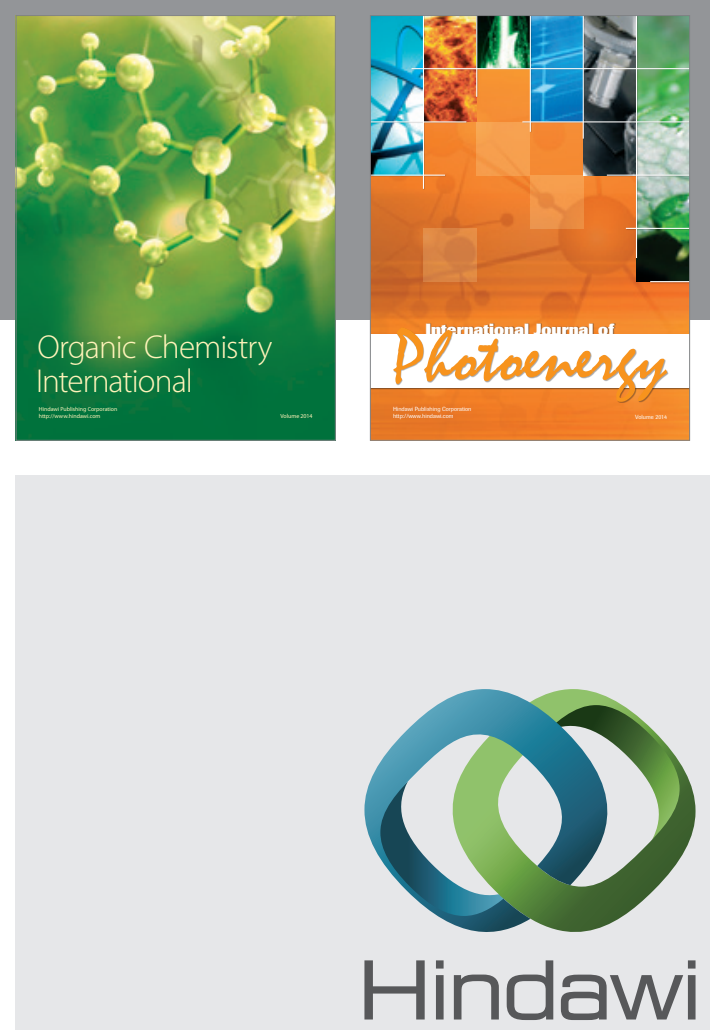

Submit your manuscripts at

http://www.hindawi.com
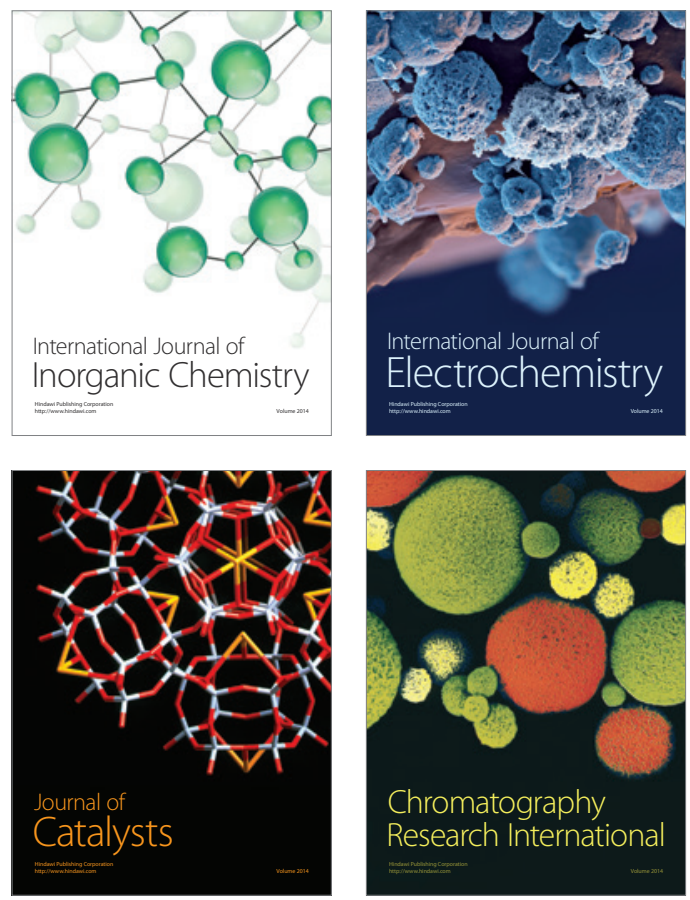
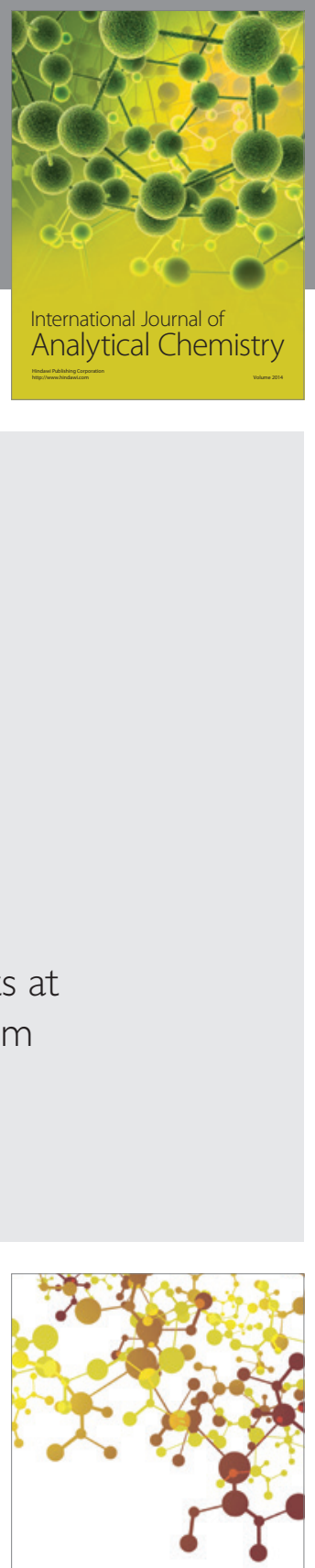

Journal of

Applied Chemistry
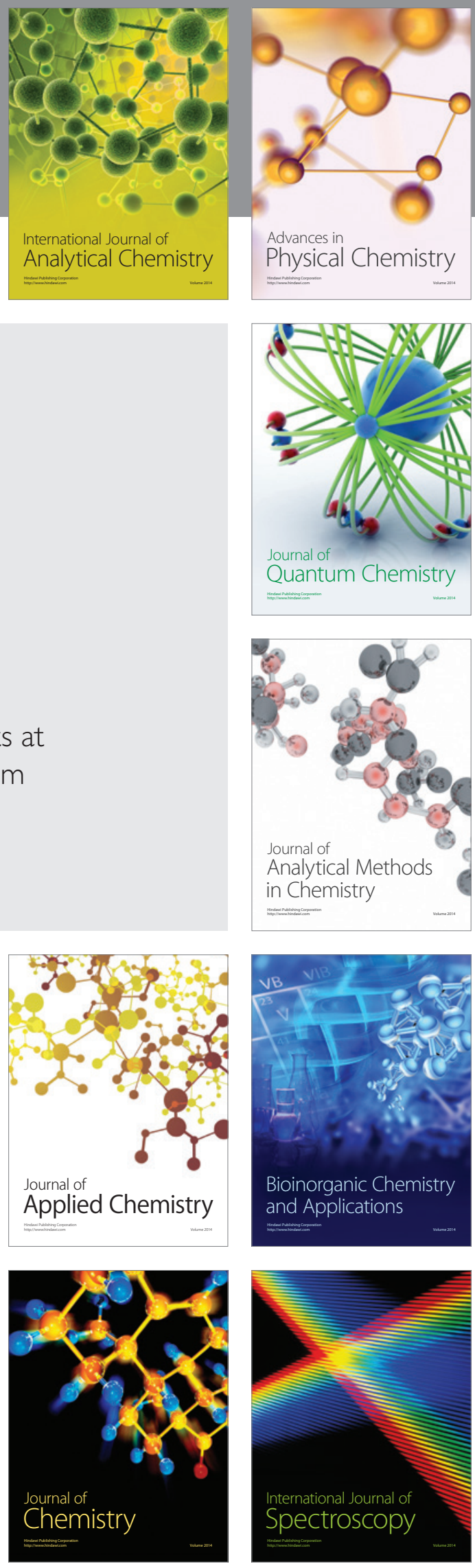